\title{
Evaluation of MAC’s Distributed Queuing Algorithm for Internet of Things Applications
}

\author{
Mabruka Mohamed Agel, Mohamed Hadi Habaebi, Sameha Alnahdi, Md Rafiqul Islam \\ Department of Electrical and Computer Engineering, International Islamic University Malaysia, Malaysia
}

\section{Article Info \\ Article history: \\ Received Jan 9, 2018 \\ Revised Mar 20, 2018 \\ Accepted Apr 4, 2018 \\ Keywords: \\ Internet of things \\ Long term evolution \\ Distributed queuing \\ M2M \\ Medium access layer}

\begin{abstract}
Internet of Things and Machine-to-Machine communications have contributed significantly on our daily life. Nevertheless, from the networking aspect they introduced a huge challenge for the performance and quality of services of networks. In this paper, a Distributed Queuing Access for LTE protocol was analyzed to enhance the random access performance for Machine to machine (M2M) applications. The M2M communications presented countless solicitations that can over-burden the access channel of the present LTE network. This prompts to an expansion in the collision probability and results in a noteworthy access delay. The introduced protocol does improve the performance of Medium access layer in terms of blocking probability, access delay, energy utilization and number of re-transmissions attempts in comparison with the standard LTE protocol without altering the existing LTE outline structure. The new protocol is intended to ensure that the random access procedure of the ordinary UE gadget will not be affected bringing about consistent implementation for the proposed convention.
\end{abstract}

Copyright (c) 2018 Institute of Advanced Engineering and Science. All rights reserved.

\section{Corresponding Author:}

Mohamed Hadi Habaebi, Department of Electrical and Computer Engineering, International Islamic University Malaysia, Jalan Gombak, Kuala Lumpur, 53100, WP, Malaysia. Email: habaebi@iium.edu.my

\section{INTRODUCTION}

Internet of Things can change the world as we are aware of it. It includes methods for enhancing the vision of the operations of industry and society by empowering remote communication component between devices, machines and smart utilization of the information to be transmitted. Internet of Things (IOT) is concerned with the automation of procedures to decrease costs, make new technologies and improve innovations. Advancements in the technology sector can profit other industries, for example, remote telehealth applications, autonomous cars, intelligent transportation and smart home automation [1], [15-17].

Communication networks are not limited to the communications that human use. Yet, there are additionally numerous new developing systems applications that are being actualized in major cities. The utilization is contingent upon the applications and other elements, for example, the degree of security, the information's quality, energy and battery life [2].

Over years, communications' industry experienced huge transformation .One of the first protocols controlling networks was Aloha's protocol which demonstrates a straightforward communication scheme. Although the initial purpose of this advancement of this protocol to facilitate the communication of satellite networks, it is considered as one of the current MAC protocols that is well suited for the applications of Internet of Things (IoT) scenarios [3]. 


\section{LITERATURE REVIEW}

Long Term Evolution (LTE) is the current significant stride in mobile radio communications, and was presented in 3GPP Release 8. LTE utilizes orthogonal frequency division multiplexing (OFDM) as its radio access innovation, together with cutting edge antenna advances. What's more, LTE is a 4G remote innovation which operates in the $700 \mathrm{MHz}$ range that means superior performance, data access and low latency [4]. This paper studies MAC layer protocols in the context of LTE technology.

\subsection{MAC Layer's Protocols}

In the most recent years, many studies have concentrated on the outline of Medium Access Control (MAC) protocols. To be up to date with the advances in technologies, MAC techniques strategies must give adaptability and effectiveness to permit the presence of these sorts of frameworks with sensible quality and reliability [5]. ALOHA and Slotted-ALOHA protocols have been generally utilized as a part of the past as random access protocols. Yet, their low throughput (0.18 and 0.36 most extreme) and potential insecurity at substantial high loads have prompted to the presence of more compelling protocols [6].

\subsection{Aloha Protocol}

Aloha protocol was created to provide remote connections between a few stations distributed spatially. By sending data from a station to the central station over a shared channel. The central station after that re-transmits the information to another channel. Collisions can happen if more than one station were attempting to send data. At then, the central channel will broadcast a collision sign. There are two variants of Aloha. Which are conventional Aloha and slotted Aloha, which is separated in the time slots and stations to exchange their data at the start of each slot. What's more, slotted Aloha permits stations to transmit packets at any time. Retransmission in case of a collision arrangement is basically the same in both protocols [7].

Slotted Aloha and Pure Aloha are both random access algorithms. The reason for these protocols to be established is that the stations in multi-channel MAC layer can get chance to transmit their packets. The fundamental contrast amongst these two protocols is that time in the pure Aloha is fixed while the time in the slotted Aloha is variant.

\subsection{LTE Standards and Protocols}

The standardization procedure for LTE was a result of the Third Generation Partnership Project (3GPP). The LTE standard was considered as an absolutely new standard. Past components were never used unless there is a convincing purpose behind them to present. There is no prerequisite for compatibility with prior variants or coding errors, for instance, LTE will work in various range utilizing different physical layer. This is resolved through the whole LTE framework by 3GPP, which supervises the whole interface to the protocol. The terminology of LTE is as shown in Figure 1. In specific, it demonstrates the device eNodeB (base station), and the interfaces between the eNodeB and UES (End User).Furthermore, E-UTRAN is the entire system, and the official name of the standards for LTE [8].

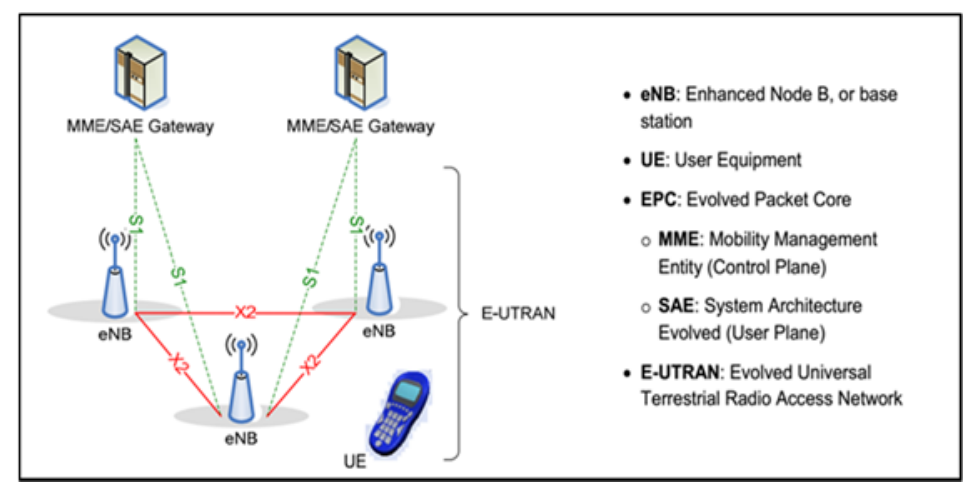

Figure 1. LTE structure and terminology [3]

\subsection{Random Access Procedure of MAC Layer in LTE}

Timing is a basic concern in random access in light of the fact that the UE can move in diverse separations from the base station and LTE requires exactness of microsecond level. Delays in arrangement speed of light alone can be the cause making a collision or a planning issue on the case that they are not kept 
up. There are two sorts of RACH: Contention based and non-contention based. The distinction is of whether there are likelihood of error in transmission utilizing the same RACH preamble [9].

\subsubsection{Contention-based random access}

Figure 2, outlines the four stages of the contention-based RACH system which are:

1. Random access preamble: a special subcarriers are dedicated in the physical layer for preambles are sent which consist of 6 bits ID.

2. Random access response: It is transmitted on Physical Downlink Control Channel (PDCCH) with a length of few TTI that carries information of UL permission, RA identifier and synchronization information.

3. Scheduled transmission: In this step, the HARQ and RLC are utilized on UL-SCH and it contains the UE identifier.

4. Contention resolution: The eNodeB utilizes this optional procedure to terminate the RACH. [10]

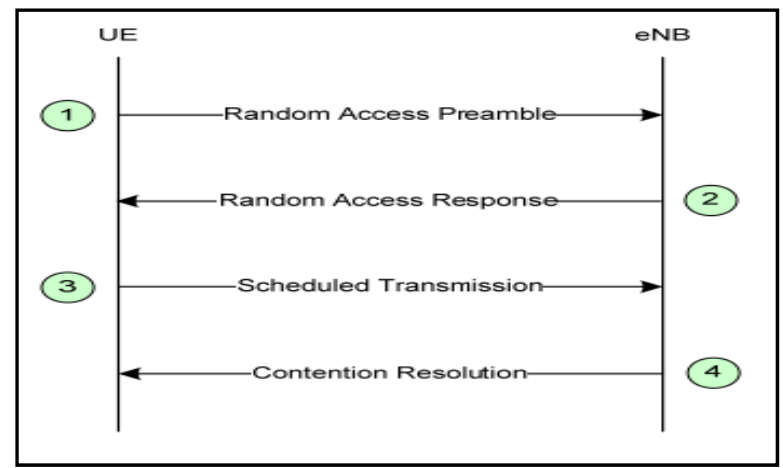

Figure 2. Contention-based RACH procedure [10]

\subsubsection{Non-Contention Based Random Access}

In the non-contention based random access strategy, there is no possibility of a preamble collision on the grounds that the code is pre-determined by the eNodeB. Figure 3 shows the three stages in this method which are:

1. Random access preamble assignment: The eNodeB relegates the 6 bit preamble code for the device.

2. Random access preamble: The UE sends the relegated preamble.

3. Random access response: Which is exactly the same for contention based random access [11].

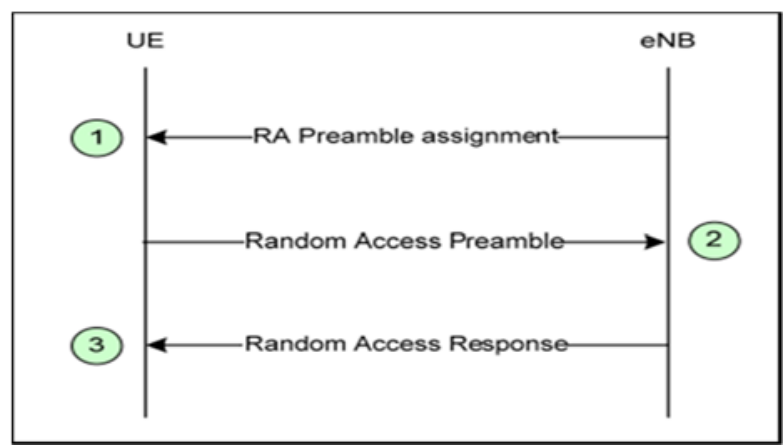

Figure 3. Non-Contention-based RACH procedure [10]

\section{RESEARCH METHOD}

The present usage of the Random Access Channel in the LTE and LTE-A is experiencing high congestion when there are tremendous quantities of gadgets attempting to get to the system. This is one of the real reasons that made the $3 \mathrm{GPP}$ accentuate the need to update the outline of the next-generation cellular networks to make it more able to bolster M2M communications. The M2M communications presented a huge 
number of access solicitations that can over-burden the access channel of the current LTE structure. This prompts to an expansion in the collision likelihood and produces a noteworthy access delay. These issues must be considered in the plan of LTE Random Access Channel (RACH) systems [12].

One of the proficient protocols, that can tackle these issues, is Distributed Queuing Random Access Protocol .The protocol puts the traffic of the recently arrived messages in the data transmission line and obstructs their transmission till the traffic turn to be light once more. In this way, the collision likelihood is diminished to an incredibly low level [2].

\subsection{Distributed Queuing}

The DQ component uses virtual distributed queue to hold the RASs for collided gadgets to retransmit the access request. The component can be actualized with straightforward alteration into the standard random access methodology as proposed by [13]. The DQ system decreases the energy consumption of MTC gadgets and the access delay while maintaining low blocking probability under high number of devices. Subsequently, the DQ approach is appropriate for the gigantic number of concurrent arrivals of MTC devices' transmissions [11].

\subsection{DQ Proposal Algorithm Using Contention Resolution Queue}

This algorithm was proposed as an arrangement by [14] for IoT applications. This simple algorithm is based on distributed queuing (DQ), which can work for an unending number of gadgets creating any traffic load. A depiction of the DQ system is given in the paper and most applicable existing investigations of DQ connected in various situations. The algorithm of 3 stages phases as shown in Figure 4.

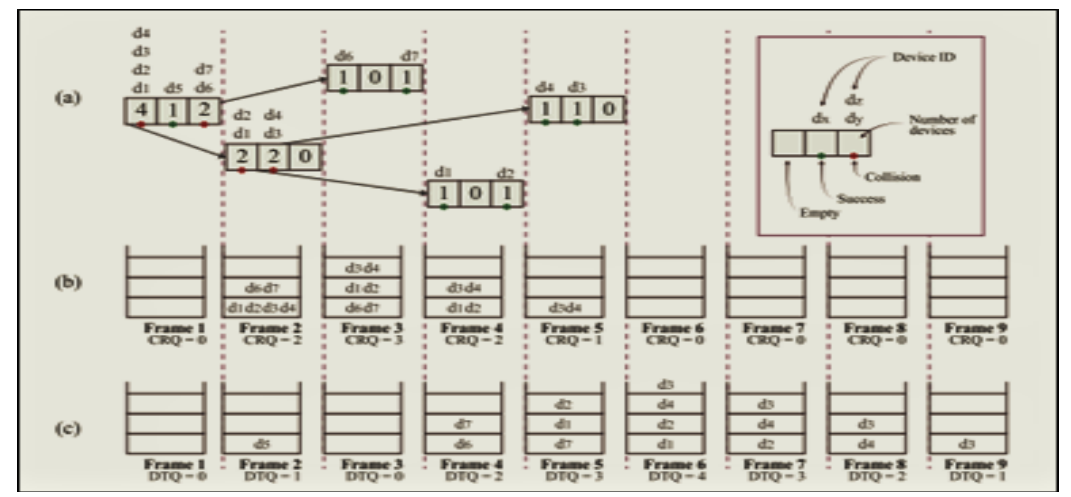

Figure 4. DQ protocol with 7 devices as proposed in [14]

\section{a. Access request sequence (ARS)}

The first stage is transmission request stage. Devices that were ideal in the previous frame send Access Request Sequence (ARS) to the coordinator using their specific slot. There are three outcomes; the first case where no device requests a transmission, the second option when only one device requests transmission in a slot and the last outcome when more than one device requests a transmission for the same slot and collision occurs.

\section{b. Contention resolution queue (CR)}

Devices that collide when requesting the same slot for transmission are moved to this queue. If collisions happen in more than one slot, the priority for retransmission request will be given to devices with the proceeding order. i.e.; devices that collide in slot 1 will be given priority for retransmission at the following frame over devices collide at slot 3. If same devices collide, again they will be put at the end of queue. When devices success in transmission request they will be moved to data transmission queue (DTQ).

\section{c. Data transmission queue (DTQ)}

Devices that do not collide at the same slot can transmit their data the next frame. The data transmission queue is according to the order of succeeding at the previous transmission order. 


\section{RESULTS AND ANALYSIS}

The performance of the Standard LTE MAC protocol analysis is validated against an analytical representation of the proposed model of [14] in NS3 and thereafter graphs are generated via MATLAB computer simulations. Four parameters were studied which are: Average access delay, Energy consumption, blocking probability and number of retransmissions.

\subsection{Access Delay}

It is characterized as the time difference between the transmission of the first preamble and the receiving of e final message by the end device. Results about average access delay are shown in Figure 5. The access delay increases as the maximum number of retransmission increases also. Without a doubt, for 56 maximum retransmissions, the outcomes of the RACH is more delayed than for the other designs; because the device spends more time in contention with more retransmission attempts.

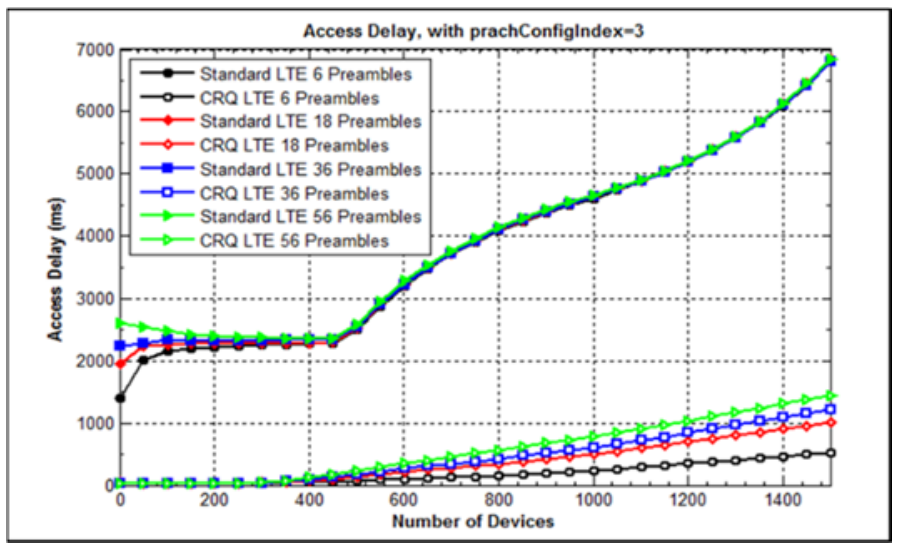

Figure 5. Access delay for PRACH=3

The graph shows also the superiority of DQ algorithm over the standard LTE. To compare the effect of Physical Random Access Channel (PRACH) value on the delay of access a performance for same configurations of preambles were used but changing the value of PRACH to 6 .

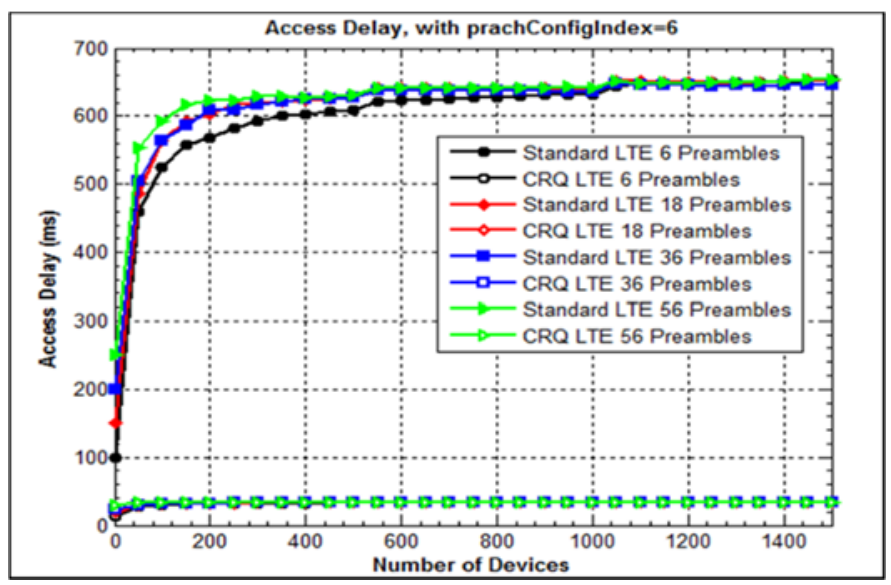

Figure 6. Access delay for PRACH=6

PRACH is used to carry random access preambles used for initiation of random access procedure. From Figure 6, it can be watched that decreasing RA slots are distributed per frame, i.e., when there are more channel access opportunities (expanding PRACH) will come about on diminishing on access delay. Furthermore, the access delay gets to has a constant value when the quantity of concurrent arrivals 
increments. As it can be noticed in both configurations, the higher the preambles dedicated the slower the access. This observation although it is similar to the finding of the paper [14] in the aspect of decreasing the access delay when applying distributed queuing algorithm, it is dissimilar on the findings of the effect of increasing the number of preambles on the delay of access. In [14] from the results the increasing of preambles will decrease the delay of access. However the findings of our simulation is consistent with those of [11], [7] and [12] which are true for the case of distributed queuing algorithm as in Figure 7.

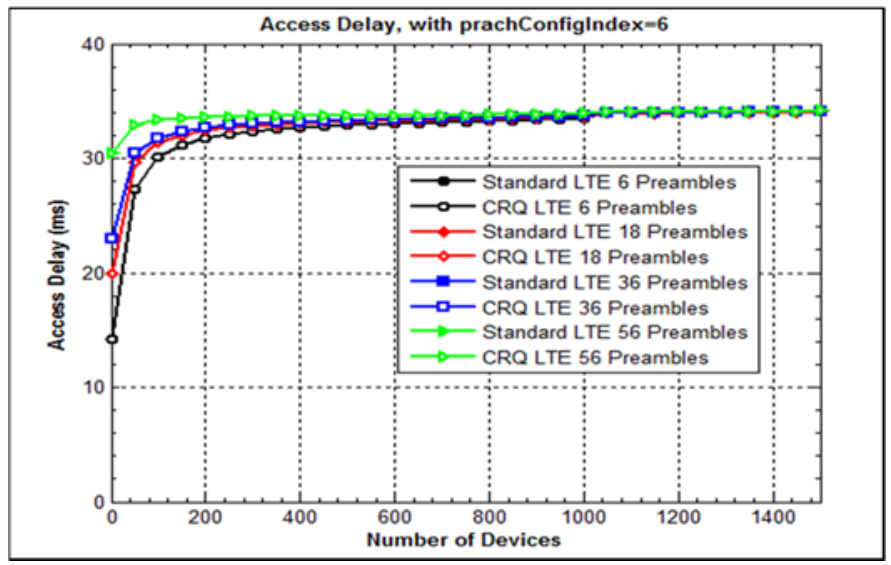

Figure 7. Access delay of DQ algorithm for PRACH=6

\subsection{Blocking probability}

It is characterized as the likelihood that a gadget achieves the maximum number of transmission and can't get to access the channel. Figures 8 shows the performance in term of blocking probability of standard LTE and DQ algorithms for PRACH=3.

From the graph it is clear that DQ algorithm performs much better than the standard LTE as the blocking probability for standard LTE is almost twice as for the proposed DQ algorithm. This is similar to the findings of [14], however it differs in terms of the effect of increasing preambles on the blocking probability as in our simulation is the higher the number of transmission preambles the less blocking probability. Our findings however are similar to those of [11], [7] and [12] in terms of increasing transmission preambles.

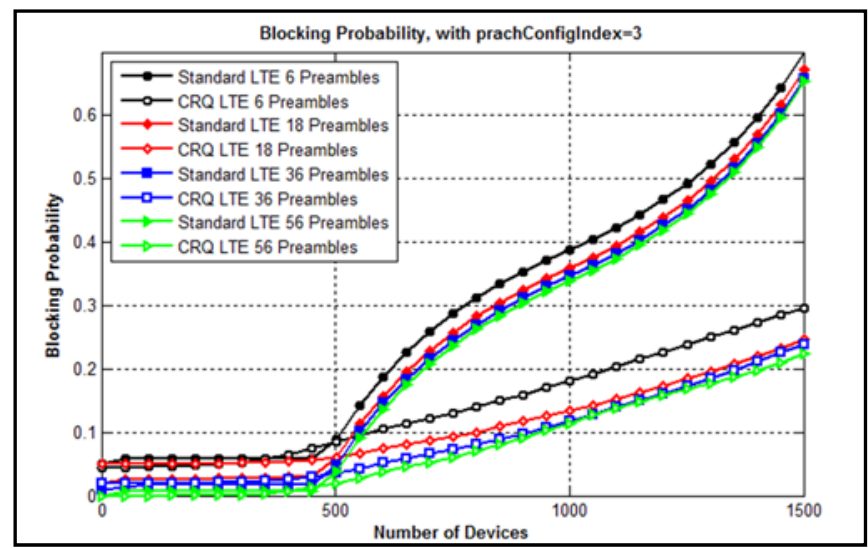

Figure 8. Blocking probability for PRACH =3

\subsection{Device Energy Consumption}

Which is characterized as the aggregate energy consumed in transmission and reception processes, from the principal RA attempt till the last successful transmission by that device in the same access. Figure 9 shows the comparison of the two models in term of energy consumption. 
At the point when the quantity of synchronous arrivals is moderately low, every one of the setups perform likewise, offering extremely productive access in energy terms. Yet, the energy rapidly increments with the quantity of synchronous arrivals, because of a higher probability of collision.

The DQ algorithm is much better than standard LTE in terms of energy saving. It can be noticed that increasing transmission preambles results in increase in energy consumption which disagrees with the findings of [14] and again it agrees with the findings of all of [11], [7] and [12].

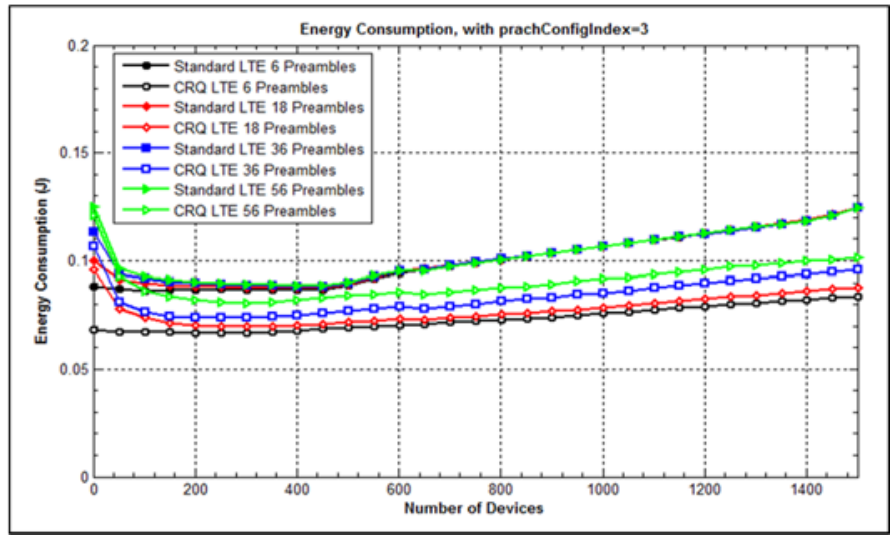

Figure 9. Energy consumption for PRACH=3

\subsection{Preamble Collision Rate}

It is characterized as measuring the mean number of preamble retransmissions required to have a successful request for transmission, this is illustrated in Figure 10. Expanding the quantity of concurrent arrivals, causes a higher probability of collision; this prompts to a higher number of required retransmissions. Additionally expanding the transmission preambles will higher the quantity of retransmission required for successful request.

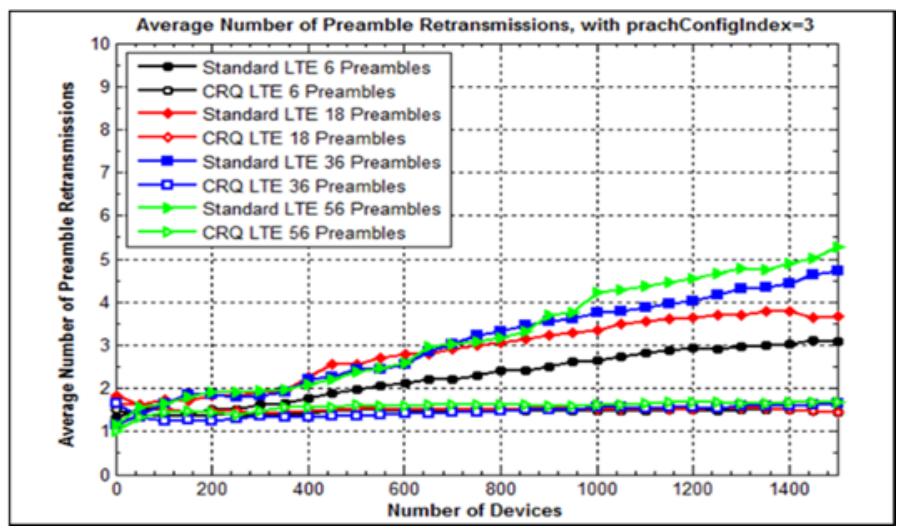

Figure 10. No. of Retransmission with PRACH=3

\section{CONCLUSION}

In this paper, multiple protocols of MAC layer were presented. The standard of LTE and its structure and terminologies where discussed. The algorithms used for MAC layer in different technologies were illustrated and a study of the effect of distributed queuing algorithms was investigated. In this paper, an evaluation of a technique proposed by paper [14] is presented in comparison with the standard LTE. The baseline performance is the number of transmission preambles of and PRACH. The two models were analyzed based on the criteria of: delay access, blocking probability, number of retransmissions and energy consumption. 
The evaluation showed that the proposed DQ algorithm outperforms the standard LTE algorithm in all basis. It also shows that increasing the number of simultaneous transmissions will increase all of delay access, blocking probability, number of retransmissions and energy consumption. Also the increasing of transmission preambles will have a negative effect on all of delay access, number of retransmissions and energy consumption, but will produce less blocking probability.

\section{ACKNOWLEDGMENT}

This work is partially sponsored by International Islamic University Malaysia research grant no. RIGS16-065-0229.

\section{REFERENCES}

[1] L. Atzori, A. Iera, and G. Morabito, "The internet of things: A survey," Computer networks, vol. 54, pp. 2787-2805, 2010.

[2] M. Beale, "Future challenges in efficiently supporting M2M in the LTE standards," in Wireless Communications and Networking Conference Workshops (WCNCW), 2012 IEEE, 2012, pp. 186-190.

[3] M. Amirijoo, P. Frenger, F. Gunnarsson, J. Moe, and K. Zetterberg, "On self-optimization of the random access procedure in $3 G$ long term evolution," in Integrated Network Management-Workshops, 2009. IM'09. IFIP/IEEE International Symposium on, 2009, pp. 177-184

[4] A. Lo, Y. W. Law, M. Jacobsson, and M. Kucharzak, "Enhanced LTE-advanced random-access mechanism for massive machine-to-machine (M2M) communications," in 27th World Wireless Research Forum (WWRF) Meeting, 2011, pp. 1-5.

[5] K. Dovelos, L. Toni, and P. Frossard, "Finite Length Performance of Random Slotted ALOHA Strategies," arXiv preprint arXiv:1607.02385, 2016.

[6] M. Noori, S. Rahimian, and M. Ardakani, "On the Capacity Region of ALOHA Protocol for the Internet of Things," arXiv preprint arXiv:1605.08806, 2016.

[7] A. Laya, L. Alonso, and J. Alonso-Zarate, "Is the Random Access Channel of LTE and LTE-A Suitable for M2M Communications? A Survey of Alternatives," IEEE Communications Surveys and Tutorials, vol. 16, pp. 4-16, 2014.

[8] 3GPP, “Radio Resource Control (RRC) - Protocol specification,” TS 36.331, V. 12.6.0, June 2015

[9] G. González, M. García-Lozano, S. Ruiz, and J. Olmos, "On the need for dynamic downlink intercell interference coordination for realistic Long Term Evolution deployments," Wireless Communications and Mobile Computing, vol. 14, pp. 409-434, 2014.

[10] T.Godfrey,” T. Long-term evolution protocol: How the standard impacts media access control”. Online dated June, 26, 2007.

[11] L. Alonso, R. Agustí, and O. Sallent, "A Near-Optimum Medium Access Control (MAC) Protocol Based on the Distributed Queueing Random Access Protocol (DQRAP) for a CDMA Mobile Communication System," IEEE Journal on Selected Areas in Communications, 1999.

[12] Samir, A., Elmesalawy, M. M., Ali, A. S., \& Ali, I. “An Improved LTE RACH Protocol for M2M Applications," Mobile Information Systems, 2016.

[13] A. Samir, M. M. Elmesalawy, A. S. Ali, and I. Ali, "An Improved LTE RACH Protocol for M2M Applications," Mobile Information Systems, vol. 2016, 2016.

[14] A. Laya, C. Kalalas, F. Vazquez-Gallego, L. Alonso, and J. Alonso-Zarate, "Goodbye, aloha!," IEEE access, vol. 4, pp. 2029-2044, 2016.

[15] Kazi Istiaque Ahmed, Mohamed Hadi Habaebi, Md Rafiqul Islam, "Enhanced Vein Detection from Video Sequences”, IJEECS, vol. 8, No.2, pp.: 420-427, November 2017.

[16] Mohamed Hadi Habaebi, Israth Jahan Chowdhury, Md Rafiqul Islam, Nur Aishah Binti Zainal, "Effects of Shadowing on LoRa LPWAN Radio Links”, IJECE, vol.7, No.6, pp.: 2970-2976, December 2017.

[17] Mohamed Habaebi, Rafhanah Rosli, Md Rafiqul Islam, "RSSI-based Human Presence Detection System for Energy Saving Automation”, IJEEI, vol. 5, no. 4, pp.: 339-350, December 2017 